\title{
ESTABILIDADE DA POLPA DE PITANGA SOB CONGELAMENTO
}

\author{
Alessandra S. LOPES ", Rafaella de A. MATTIETTO’, Hilary C. de MENEZES
}

\section{RESUMO}

Dentre as frutas tropicais, a pitanga, ainda pouco estudada, apresenta um grande apelo sensorial e uma excelente qualidade vitamínica devido ao seu elevado teor de carotenóides. Este trabalho teve como objetivo avaliar a estabilidade da polpa de pitanga preservada por congelamento $\left(-18^{\circ} \mathrm{C}\right)$ através das análises de $\mathrm{pH}$, acidez total titulável, sólidos solúveis, sólidos totais, açúcares redutores e totais e aceitação sensorial nos tempos $0,30,60,90$ dias, carotenóides totais e cor instrumental, nos tempos 0 , 15, 30, 45, 60 e 90 dias. A reação cinética da degradação do parâmetro de cor a* se ajustou melhor ao modelo cinético de 1 ordem $\left(\mathrm{R}^{2}=91,69 \%\right)$. No entanto, para a diferença total de cor $\left(\Delta \mathrm{E}^{*}\right)$ apresentou melhor adequação ao modelo de ordem $z e r o\left(\mathrm{R}^{2}=91,91 \%\right)$. $\mathrm{Na}$ análise sensorial, a a parência variou significativamente $(\mathrm{p} \leq 0,05)$ aos 90 dias de estocagem. Houve uma forte queda na aceitabilidade sensorial e na atitude positiva de compra do néctar formulado com a polpa de pitanga armazenada por 90 dias a - $18 \mathrm{C}$.

Palavras-chave: Eugenia uniflora L.; congelamento; estabilidade; cor; sensorial.

\section{SUMMARY}

STABILITY OF FROZEN PITANGA PULP. Amongst tropical fruits, the pitanga (Eugenia uniflora L.), still little studied, shows great sensory appeal and excellent vitamin quality, due to its high carotenoid level. The objective of this study was to evaluate the stability of pitanga pulp during frozen storage $\left(-18^{\circ} \mathrm{C}\right)$ with respect to the following parameters: $\mathrm{pH}$, total titratable acidity, soluble and total solids, total and reducing sugars and sensory acceptance determined after 0, 30, 60 and 90 days, and total carotenoid contents and instrumental colour measured after $0,15,30,45,60$ and 90 days. The reaction kinetics for the degradation of the colour parameter $\mathrm{a}^{*}$ adjusted better to a $1^{*}$ order kinetic model $\left(\mathrm{R}^{*}=91.69 \%\right)$, whereas the total difference in colour $\left(\Delta \mathrm{E}^{*}\right)$ adjusted better to a zero order kinetic model $\left(\mathrm{R}^{2}=91.91 \%\right)$. In the sensory analysis, the attribute of appearance varied significantly ( $\left.\leq 0.05\right)$ after 90 days of frozen storage. Thus there was a marked fall in sensory acceptance and in the positive attitude towards buying of the nectar formulated with pitanga pulp stored for 90 days at $-18^{\circ} \mathrm{C}$

Keywords: Eugenia uniflora L.; frozen; stability; colour; sensory analysis.

\section{1 - INTRODUÇÃO}

A pitanga (Eugenia uniflora L.) é um fruto tropical nativo das regiões Sul e Sudeste do Brasil e que tem se adaptado favoravelmente às condições climáticas e edáficas da região Nordeste $[10,18]$. Dentre os frutos existentes na natureza, a pitanga figura entre os que possuem os maiores teores de carotenóides totais $(225,9 \mu \mathrm{g} / \mathrm{g})$ [5].

No Brasil, não se conhecem variedades perfeitamente definidas de pitangueiras, e é comum encontrar frutos de coloração que variam desde o alaranjado, vermelho escuro até o roxo $[13,14]$.

As pitangas, quando maduras, são facilmente vulneráveis à depreciação, sendo de dificil conservação, o que dificulta o seu transporte e comercialização a grandes distâncias [13]. A polpa de fruta constitui uma alternativa de consumo para os mercados nacionais ou internacionais, mesmo em períodos fora da safra de produção.

Recebido para publicação em 04/02/2005. Aceito para publicação em 11/08/2005 (001470).

Departamento de Tecnologia de Alimentos, Faculdade de Engenharia de Alimentos/Universidade Estadual de Campinas. Caixa Postal: 6121. CEP: 13083-970, Campinas-SP-Brasil. E-mail: aled@fea.unicamp.br/hilary@fea.unicamp.br.

A quem a correspondência deve ser enviada.
O congelamento é um dos processos mais indicados para a preservação das propriedades químicas, nutricionais e sensoriais de polpas de frutas, no entanto, apresenta custos de produção, transporte e armazenamento relativamente elevados. Os microrganismos não são considerados um grande problema em alimentos congelados, pois estes não crescem em temperaturas usuais de congelamento $\left(-18^{\circ} \mathrm{C}\right)$. No entanto, a ação das enzimas é preocupante, pois pode provocar significativas alterações de cor e sabor em polpas de frutas congeladas [9].

A estabilidade dos carotenóides difere bastante nos alimentos, mesmo quando submetidos a processamento e condições de estocagem idênticas. A principal causa de destruição dos carotenóides é a oxidação (enzimática ou não-enzimática). O processo de congelamento, especialmente o congelamento rápido, e a estocagem sob temperaturas de congelamento geralmente propiciam a retenção dos carotenóides nos alimentos [22].

A vida-de-prateleira de um alimento é o tempo em que ele pode ser conservado em determinadas condições de temperatura, umidade relativa, etc, sofrendo pequenas, mas bem estabelecidas alterações consideradas aceitáveis pelo fabricante, pelo consumidor e pela legislação vigente. Assim, é necessário o uso da análise sensorial para avaliar a vida-de-prateleira dos alimentos [8].

A análise sensorial possui várias aplicações, tais como: controle e garantia da qualidade, desenvolvimento de novos produtos, testes de consumidores, estudos de percepção humana, correlação com medidas fisicas, químicas, instrumentais [7]. 
Como garantia de qualidade, os testes sensoriais são muito eficientes, já que são medidas multidimensionais integradas, possuindo importantes vantagens, tais como: ser capaz de identificar a presença ou ausência de diferenças perceptiveis, definir características sensoriais importantes de um produto de forma rápida, e ser capaz de detectar particularidades que não podem ser detectadas por outros procedimentos analíticos [20].

Este estudo objetivou avaliar a estabilidade da polpa de pitanga congelada durante um período de estocagem de 90 dias, através da monitoração de $\mathrm{pH}$, acidez total titulável, sólidos solúveis, sólidos totais, açúcares redutores e totais e aceitação sensorial nos tempos 0, 30, 60, 90 dias, carotenóides totais e cor instrumental, avaliados nos tempos $0,15,30,45,60$ e 90 dias.

\section{2 - MATERIAL E MÉTODOS}

\section{1 - Material}

Polpa de pitanga $(6,5 \mathrm{~kg})$ obtida do despolpamento de frutos in natura de coloração avermelhada provenientes de Valinhos (SP), fracionada $(0,5 \mathrm{~kg})$ e acondicionada em sacos plásticos retangulares de polietileno de baixa densidade com dimensões de $34 \times 24,5 \mathrm{~cm}$ (comprimento $\mathrm{x}$ largura) e espessura média de $0,117 \pm 0,001 \mathrm{~mm}$, considerando as duas faces da embalagem.

As embalagens previamente seladas foram submetidas ao processo de congelamento rápido em congelador de placas marca FRIGOSTRELLA DO BRASIL, mod. P.M5 por 3 horas $\left(30^{\circ} \mathrm{C}\right)$ e armazenadas em câmara de estocagem $\left(-18^{\circ} \mathrm{C}\right)$.

\section{2 - Análises}

2.2.1. Análises físico-químicas da polpa de pitanga congelada

- $\quad$ pH: segundo método $\mathrm{n}^{\circ} 981.12$ da AOAC [2];

- Acidez Total Titulável: segundo método no 942.15 da AOAC [2];

- Sólidos Solúveis (como ${ }^{\circ} \mathrm{Brix}$ ): baseado na leitura direta dos graus Brix da amostra a $20^{\circ} \mathrm{C}$ em refratômetro digital da marca LEICA, mod. AR200;

- Sólidos Totais: realizado em analisador de umidade por infra-vermelho da marca GEHAKA, mod IV 2002.

- Açúcares Redutores e Totais: método n³1.034-6 da AOAC [1];

- Carotenóides Totais: segundo método descrito por RODRIGUEZ-AMAYA [22];

- Determinação Instrumental de Cor: a cor foi avaliada através de um espectrofotômetro Colorquest Hunterlab. O sistema de leitura utilizado foi o CIELAB, representado pelos seguintes parâmetros: coordenada $\mathrm{L}^{*}$ (luminosidade), coordenada de cromaticidade $a^{*}$ (-a verde, +a vermelho) e a coordenada de cromaticidade $b^{*}(-\mathrm{b}$ azul, $+\mathrm{b}$ amarelo). As amostras foram colocadas em cubeta de vidro opticamente limpo com $10 \mathrm{~mm}$ de caminho óptico. Foi calculada a diferença total de cor $\left(\Delta \mathrm{E}^{*}\right)$ de acordo com a equação abaixo (Equação 1):

$\Delta \mathrm{E}^{*}=\left[\left(\Delta \mathrm{L}^{*}\right)^{2}+\left(\Delta \mathrm{a}^{*}\right)^{2}+\left(\Delta \mathrm{b}^{*}\right)^{2}\right]^{1}$

(Equação1)

onde: $\Delta$ é a diferença entre cada parâmetro de cor da amostra no tempo zero (padrão) e amostra armazenada a $-18^{\circ} \mathrm{C}$ nos tempos $15,30,45,60$ e 90 dias.

\subsection{2 - Análise sensorial}

A aceitabilidade da polpa de pitanga congelada foi avaliada por uma mesma equipe de 40 provadores, consumidores de sucos e néctares de frutas tropicais, recrutados dentre alunos, funcionários e professores da Faculdade de Engenharia de Alimentos (UNICAMP). A polpa de pitanga foi avaliada na forma de um néctar com 20 Brix (50\% de polpa de pitanga, 50\% de água filtrada), aplicando-se os testes de aceitação em relação à aparência, aroma e sabor com escala hedônica não-estruturada e atitude de compra $(5=$ certamente compraria, $3=$ tenho dúvidas se compraria ou não, $1=$ certamente não compraria) $[17,24]$. A amostra foi apresentada em cabine individual, a temperatura de refrigeração $\left(10^{\circ} \mathrm{C}\right)$ e em copos plásticos descartáveis de $50 \mathrm{~mL}$, codificados com algarismos de três dígitos.

Os resultados dos testes de aceitação sensorial foram analisados por Análise de Variância (ANOVA) e teste de médias de Tukey ( $\mathrm{p} \leq 0,05)$, com auxilio do programa Statistica ${ }^{\circledR}$ versão 5.0 [23]. Os resultados dos testes de intenção de compra foram avaliados através da freqüência das notas dadas pelos provadores.

\subsection{3 - Análise estatística dos resultados}

Todas as análises fisico-químicas foram realizadas em triplicata, e os valores obtidos foram avaliados com o auxílio do programa Statistica ${ }^{\circledR}$ versão 5.0 empregando as seguintes metodologias estatísticas:

- Análise de variância (ANOVA) ao nivel de 5\% de significância estatística segundo o teste $\mathrm{F}$;

- $\quad$ Teste de Tukey $(\mathrm{p} \leq 0,05)$;

- Análise de regressão.

\section{3 - RESULTADOS E DISCUSSÃO}

\section{1 - Caracteristicas fisico-quimicas da polpa de pitanga congelada}

Na Tabela 1 são mostrados os resultados das análises físico-químicas realizadas durante 90 dias de estocagem da polpa de pitanga $\left(-18^{\circ} \mathrm{C}\right)$.

Os valores de $\mathrm{pH}$ (Tabela 1) obtidos nos tempos 0,30 , 60 e 90 variaram de 3,27 a 3,40 e atenderam aos padrões de identidade e qualidade legais vigentes para $\mathrm{pH} \mathrm{2,5} \mathrm{a}$ 3,4 em polpa pitanga [4]. 
TABELA 1 - Caracterização físico-química da polpa de pitanga congelada durante estocagem

\begin{tabular}{|c|c|c|c|c|}
\hline \multirow[t]{2}{*}{ Parâmetro } & \multicolumn{4}{|c|}{ Tempo de estocagem (dias) } \\
\hline & 0 & 30 & 60 & 90 \\
\hline $\mathrm{pH}$ & $3,27 \pm 0,01^{c}$ & $3,39 \pm 0,01^{b}$ & $3,40 \pm 0,01^{\mathrm{a}}$ & $3,40 \pm 0,00^{a}$ \\
\hline $\begin{array}{l}\text { Acidez Total } \\
\text { Titulável }(\mathrm{g} \text { ácido } \\
\text { cítrico } / 100 \mathrm{~g})\end{array}$ & $1,24 \pm 0,01^{a}$ & $1,25 \pm 0,01^{a}$ & $1,24 \pm 0,02^{a}$ & $1,25 \pm 0,01^{a}$ \\
\hline $\begin{array}{l}\text { Sólidos Solúveis } \\
\text { ( }{ }^{\circ} \text { Brix) }\end{array}$ & $11,47 \pm 0,06^{\mathrm{a}}$ & $10,97 \pm 0,06^{b}$ & $10,90 \pm 0,00^{b}$ & $10,73 \pm 0,06$ \\
\hline Sólidos Totais (\%) & $12,29 \pm 0,14^{a}$ & $12,28 \pm 0,04^{a}$ & $12,20 \pm 0,12^{\mathrm{a}}$ & $12,20 \pm 0,02$ \\
\hline $\begin{array}{l}\text { Açúcares Redutores } \\
\text { (g glicose/100g) }\end{array}$ & $7,65 \pm 0,11^{\mathrm{a}}$ & $7,42 \pm 0,17$ a & $7,54 \pm 0,24 \mathrm{a}$ & $7,73 \pm 0,05^{\mathrm{a}}$ \\
\hline $\begin{array}{l}\text { Açúcares Totais } \\
\text { (g glicose / 100g) }\end{array}$ & $7,77 \pm 0,22^{\mathrm{a}}$ & $7,99 \pm 0,38^{a}$ & $7,93 \pm 0,19^{a}$ & $7,90 \pm 0,12^{\mathrm{a}}$ \\
\hline
\end{tabular}

GUIMARÃES et al. [11] observaram um aumento do $\mathrm{pH}$ da polpa de pitanga preservada a $-18^{\circ} \mathrm{C}$ de 2,3 para 1,9 após 150 dias de armazenamento, sendo este comportamento divergente ao encontrado no presente trabalho.

A acidez total titulável da polpa de pitanga não apresentou diferença estatística $(\mathrm{p}>0,05)$ durante os 90 dias de estocagem (Tabela 1), encontrando-se em acordo com a legislação vigente que estabelece um limite mínimo de 0,92g ácido cítrico/100g [4].

A legislação vigente determina o limite mínimo de $7 \mathrm{~g} / 100 \mathrm{~g}$ de sólidos totais na polpa de pitanga. Este limite estabelece um padrão de qualidade para polpas de frutas comercializadas, e objetiva evitar a fraude por diluição com água. Como pode ser visto na Tabela 1, o teor dos sólidos totais se encontrou dentro dos parâmetros requisitados pela legislação em vigor durante os 90 dias de estocagem a $-18^{\circ} \mathrm{C}$.

De acordo com a Tabela 1, os teores de açúcares redutores e totais não diferiram estatisticamente $(p>0,05)$ durante estocagem por 90 dias a $18^{\circ} \mathrm{C}$.

Diante dos resultados mostrados na Tabela 1, observou-se que apesar de haverem pequenas variações nas características físico-químicas da polpa de pitanga armazenada a $-18^{\circ} \mathrm{C}$ por 90 dias, estas não ultrapassaram os limites determinados pela lei em vigor [4].

MÉLO, LIMA \& NASCIMENTO [19] investigaram a influência da temperatura no armazenamento de frutos de pitanga e observaram alterações significativas $(\mathrm{p} \geq 0,05)$ nos valores de $\mathrm{pH}$, acidez total titulável, sólidos solúveis e totais a partir do $10^{\circ}$ dia de estocagem sob temperatura de $-18^{\circ} \mathrm{C}$. O congelamento de pitangas maduras e semimaduras alterou intensamente a textura e as características físico-quimicas dos frutos e, por isso, os mesmos apresentaram destino mais adequado ao processamento industrial.

O armazenamento da pitanga na forma de polpa em temperatura de congelamento é a alternativa mais viável do ponto de vista da qualidade do produto.

\section{2 - Carotenóides totais}

De acordo com a Tabela 2, observou-se que ocorreu uma queda significativa do teor de carotenóides totais nos primeiros 30 dias de estocagem, no entanto, nos tempos 45, 60 e 90 dias não foi verificado decréscimo importante.

Comportamento similar foi encontrado para a degradação das antocianinas da polpa de pitanga de coloração roxa estocada sob congelamento por 360 dias. Houve um decréscimo significativo das antocianinas $(8,77 \%)$, após 60 dias de estocagem, entretanto o teor desse pigmento manteve-se praticamente constante nos meses subseqüentes [15].

TABELA 2 - Carotenóides totais da polpa de pitanga durante estocagem por 90 dias a $-18^{\circ} \mathrm{C}$

\begin{tabular}{ccc}
\hline $\begin{array}{c}\text { Tempo de } \\
\text { estocagem (dias) }\end{array}$ & $\begin{array}{c}\text { Carotenóides totais } \\
(\mu \mathrm{g} / \mathrm{g})^{*}\end{array}$ & $\begin{array}{c}\text { Retenção de } \\
\text { carotenóides } \\
\text { totais }(\%)\end{array}$ \\
\hline 0 & $123,73 \pm 1,17^{\mathrm{a}}$ & 100 \\
15 & $117,14 \pm 1,25^{\mathrm{b}}$ & 94,68 \\
30 & $106,71 \pm 0,30^{\mathrm{d}}$ & 86,24 \\
45 & $109,67 \pm 0,89^{\mathrm{c}}$ & 88,64 \\
60 & $107,04 \pm 1,22^{\text {cd }}$ & 86,50 \\
90 & $106,71 \pm 0,77^{\mathrm{d}}$ & 86,24 \\
\hline
\end{tabular}

Valores representam médiatdesvio padrão

${ }^{*} \mu \mathrm{g}$ referente ao licopeno $/ \mathrm{g}\left(\mathrm{A}^{1 \kappa_{1 \mathrm{~cm}}}\right.$ licopeno em éter de petróleo $\left.=3450\right)$. $a, b, c, d$ Letras diferentes em uma mesma linha indicam diferença estatística (p $\leq 0,05)$.

Os carotenóides estão naturalmente protegidos nos tecidos das plantas, no entanto, quando frutas e vegetais são cortados ou desintegrados ocorre um aumento da exposição dos carotenóides ao oxigênio e contato com enzimas que catalisam o processo de oxidação [21].

A maior razão de perda dos carotenóides é a oxidação e muitos fatores, tais como exposição à luz e ao oxigênio, tipo de matriz alimentícia, presença de enzimas, disponibilidade de água e presença de antioxidantes e/ou próoxidantes podem influenciar o processo oxidativo [5, 22].

O processamento e a estocagem dos alimentos podem alterar significativamente a composição qualitativa e quantitativa dos carotenóides [5,6]. A retenção dos carotenóides pró-vitamínicos durante a estocagem de alimentos processados é favorecida pela baixa temperatura, proteção da luz, exclusão do oxigênio (por vácuo, enchimento à quente, atmosfera modificada ou embalagem impermeável ao oxigênio) e antioxidantes, presentes naturalmente ou adicionados como meio de preservação do alimento [21].

No estudo de quantificação de carotenóides totais em duas seleções de pitangas de colorações distintas, roxa e vermelha, foram encontrados valores de $111 \pm 2 \mu \mathrm{g} / \mathrm{g}$ e $104 \pm 0 \mu \mathrm{g} / \mathrm{g}$, respectivamente [14].

Na avaliação dos carotenóides totais em polpa de pitanga não branqueada e estocada por 90 dias a $-18^{\circ} \mathrm{C}$ foram observados decréscimos consideráveis devido à oxi- 
dação enzimática [6].

THAKUR \& ARYA [26] verificaram que houve uma maior perda dos carotenóides em polpas de manga que não foram submetidas a um tratamento térmico (branqueamento) preliminar à estocagem em temperatura de $12 \mathrm{C}$ e relataram a necessidade da inativação das enzimas responsáveis pelo processo oxidativo.

Carotenóides como o $\alpha$-caroteno e $\beta$-caroteno representam cerca de 80 a $90 \%$ do total de carotenóides presentes em cenouras, que após oito semanas de estocagem a 1 C apresentaram um decréscimo de $30 \%$ desses pigmentos [18].

\section{3 - Cor instrumental}

Como pode ser visto na Tabela 3, houve decréscimo dos parâmetros $\mathrm{L}^{*}, \mathrm{a}^{*} \mathrm{e} \mathrm{b}^{*} \mathrm{e}$ aumento de $\Delta \mathrm{E}^{*}$ ao longo do tempo de estocagem. Após os 90 dias de estocagem, observou-se que houve uma maior queda média de $a^{*}$ $(16,1 \%)$, seguido de $b^{*}(11,1 \%)$ e $L^{*}(5,2 \%)$. Desta forma, é confirmada a importância da coordenada de cromaticidade $a^{*}$ no estudo de estabilidade da polpa de pitanga, visto que, este parâmetro está diretamente relacionado aos carotenóides presentes nesta fruta.

TABELA 3 - Parâmetros de cor $\left(\mathrm{L}^{*}, \mathrm{a}^{*} \mathrm{e} \mathrm{b}^{*}\right)$ e diferença total de $\operatorname{cor}\left(\Delta \mathrm{E}^{*}\right)$ em relação ao padrão (tempo 0) da polpa de pitanga durante estocagem por 90 dias a $-18 \mathrm{C}$

\begin{tabular}{|c|c|c|c|c|}
\hline $\begin{array}{c}\text { Tempo de } \\
\text { estocagem } \\
\text { (dias) }\end{array}$ & $L^{\star}$ & $a^{*}$ & $b^{*}$ & $\Delta \mathrm{E}^{*}$ \\
\hline 0 & $37,54 \pm 0,03^{a}$ & $20,67 \pm 0,05^{a}$ & $15,62 \pm 0,11^{\mathrm{a}}$ & - \\
\hline 15 & $36,96 \pm 0,02^{\mathrm{b}}$ & $20,12 \pm 0,04^{b}$ & $15,01 \pm 0,02^{\mathrm{b}}$ & $1,00 \pm 0,07$ \\
\hline 30 & $36,67 \pm 0,03^{c}$ & $19,32 \pm 0,10^{c}$ & $14,56 \pm 0,19 c$ & $1,92 \pm 0,08^{b}$ \\
\hline 45 & $36,19 \pm 0,03^{d}$ & $18,20 \pm 0,06^{d}$ & $\underset{\mathrm{d}}{14,33 \pm 0,03 \mathrm{c}}$ & $3,09 \pm 0,03^{c}$ \\
\hline 60 & $35,85 \pm 0,12^{e}$ & $17,54 \pm 0,07^{e}$ & $\underset{e}{14,09 \pm 0,06 \mathrm{~d}}$ & $3,87 \pm 0,08$ \\
\hline 90 & $35,60 \pm 0,05^{f}$ & $17,35 \pm 0,02^{e}$ & $13,89 \pm 0,04^{e}$ & $4,18 \pm 0,02^{e}$ \\
\hline
\end{tabular}

$a, b, c, d, d, f$, Letras diferentes em uma mesma linha indicam diferença estatística (ps0,05).

Para a determinação do modelo cinético da variação de cor instrumental da polpa de pitanga congelada foram utilizados os parâmetros de cor $\mathrm{a}^{*}$ e $\Delta \mathrm{E}^{*}$, segundo as equações 2 e 3, que representam as reações de zero e primeira ordem, respectivamente. Para a reação de ordem zero a taxa de alteração é constante com o tempo, enquanto que em uma reação de primeira ordem esta taxa apresenta decréscimo exponencial [9].

$$
\begin{aligned}
& A=A_{O}-k t \\
& A=A_{O} \exp ^{(-k t)}
\end{aligned}
$$

(Equação 2)

(Equação 3)

onde:

Ao = fator de qualidade inicial; $A$ = fator de qualidade no tempo decorrido; $\mathrm{k}$ = constante da reação; $\mathrm{t}=$ tempo decorrido.
De acordo com TAOUKIS, LABUZA \& SAGUY [25] e LABUZA [12], duas diferentes abordagens devem ser usadas alternativamente para definir o modelo cinético de uma reação. A primeira envolve o uso dos dados diretos obtidos experimentalmente, e requer idealmente alterações do parâmetro avaliado de $75 \%$, ou um mínimo de $50 \%$. A segunda abordagem trata de reações de pequeno grau de conversão (5\%), e neste caso, faz-se necessário o tratamento dos dados usando-se a razão do parâmetro em relação ao valor inicial.

Desta forma, a variação da coordenada a* para a polpa de pitanga congelada ao longo do tempo de estocagem foi avaliada segundo os modelos de zero (Figura 1) e primeira ordem linearizado (Figura 2).

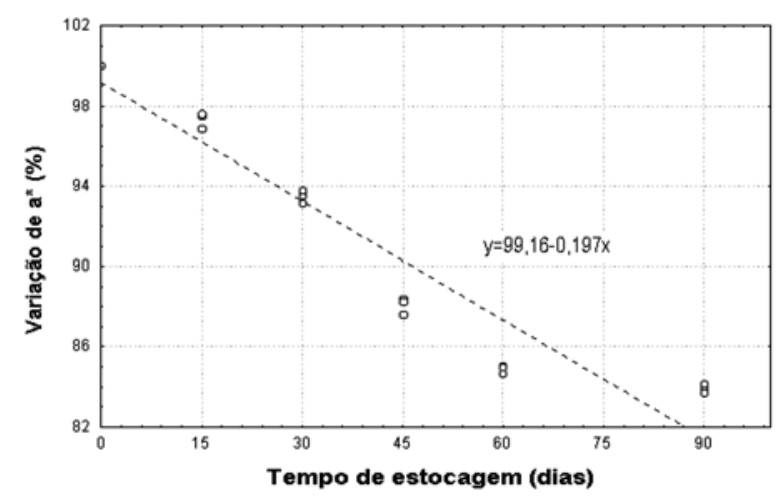

FIGURA 1 - Modelo cinético de ordem zero da variação do parâmetro de cor $a^{*}$ na polpa de pitanga congelada

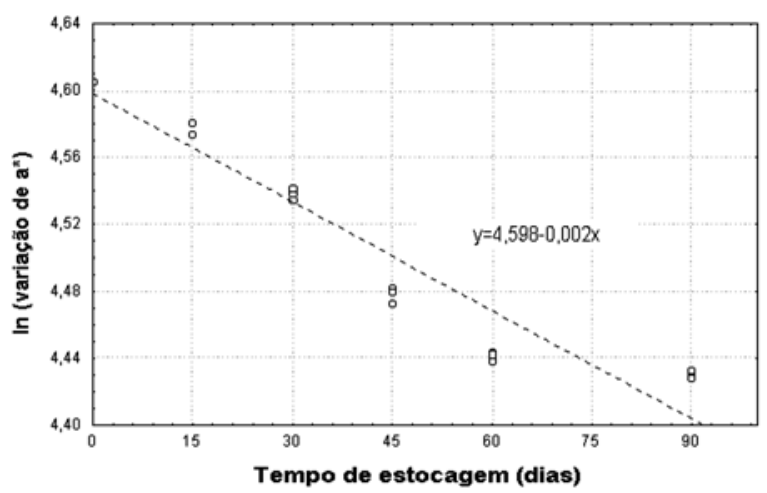

FIGURA 2 - Modelo cinético de primeira ordem da variação do parâmetro de cor $a^{*}$ na polpa de pitanga congelada

Os critérios utilizados para avaliar o modelo cinético de reação foram: coeficiente de determinação $\left(R^{2}\right)$ e o desvio relativo médio $(\mathrm{P})$ (Equação 4). Um valor de $\mathrm{P}$ menor que cinco corresponde a um bom ajuste da equação aos dados experimentais [16]. 


$$
P=\frac{100}{n} \sum_{i=1}^{n} \frac{\mid\left(V^{(0 b s)} i-V^{(p r e d s)}\right)}{V^{(o b s)_{i}}}
$$

onde: $\mathrm{n}=\mathrm{n}$ de observações;

$\mathrm{V}^{(0,0)}=$ valor observado experimentalmente;

$\mathrm{V}^{\mathrm{nn} \text { real }}=$ valor predito pelo modelo de regressão linear.

O coeficiente de determinação $\left(R^{2}\right)$ da regressão linear, em muitos casos, é suficiente para a determinação da ordem aparente de reação e da equação de melhor ajuste. Ao mesmo tempo, deve-se ter cuidado quando a alteração estudada é menor que $50 \%$, pois pode não haver diferenciação significativa dos ajustes aos modelos cinéticos de zero ou primeira ordem. Por outro lado, se o término da vida-de-prateleira referir-se a uma conversão de $20 \%$, ambos os modelos serão válidos [25].

TABELA 4 - Parâmetros cinéticos e estatísticos da variação de $a^{*}$ na polpa de pitanga congelada

\begin{tabular}{ccccc}
\hline $\begin{array}{c}\text { Ordem de reação } \\
\text { aparente }\end{array}$ & \multicolumn{2}{c}{$\begin{array}{c}\text { Parâmetros } \\
\text { cinéticos }\end{array}$} & \multicolumn{2}{c}{$\begin{array}{c}\text { Parâmetros } \\
\text { estatisticos }\end{array}$} \\
\cline { 2 - 5 } & $\mathrm{k}\left(\right.$ dia $\left.^{-1}\right)$ & $\mathrm{t}_{1 / 2}$ (dias) & $\mathrm{R}^{2}(\%)$ & $\mathrm{P}(\%)$ \\
\hline 0 & 0,197 & 251,68 & 91,23 & 1,79 \\
1 & 0,002 & 346,57 & 91,69 & 0,37 \\
\hline
\end{tabular}

Os valores de $\mathrm{R}^{2}$ são muito próximos para ambos os modelos, no entanto, observa-se uma diferenciação melhor em relação ao valor do desvio relativo médio (P). Segundo este último critério, a variação de $a^{*}$ da polpa de pitanga congelada é mais bem ajustada ao modelo de primeira ordem (Tabela 4).

O tempo de meia-vida $\left(\mathrm{t}_{1 / 2}\right)$ é definido como o tempo necessário para uma redução de $50 \%$ do valor inicial do parâmetro avaliado. Para as reações da variação da coordenada de cor instrumental a* na polpa de pitanga congelada, os tempos de meia-vida para os modelos de zero e primeira ordem foram 251,7 e 346,6 dias, respectivamente. As constantes de reação de zero e primeira ordem, obtidas através de regressão linear, foram de 0,197 e 0,002 dia , respectivamente (Tabela 4).

Na análise de regressão é também fundamental o exame dos gráficos de resíduos para que se possa determinar qual modelo é o mais adequado [3].

Verificou-se que o ajuste de primeira ordem apresenta maior aleatorização dos resíduos, e estes estão mais próximos do eixo do tempo $(\mathrm{y}=0)$. No ajuste de ordem zero, não se observa uma distribuição aleatória dos resíduos, ou seja, há uma concentração de resíduos positivos, nos tempos 0,15 e 30 dias, e negativos, nos tempos 45 e 60 dias (Figura 3).

O modelo cinético referente à diferença total de cor (Figura 4) da polpa de pitanga armazenada a $18^{\circ} \mathrm{C}$ durante 90 dias foi mais bem ajustado à equação de ordem zero. O valor do coeficiente de determinação $\left(R^{2}\right)$ obtido foi de $91,91 \%$, e a constante da velocidade de reação $(\mathrm{k})$ foi 0,049 dia.

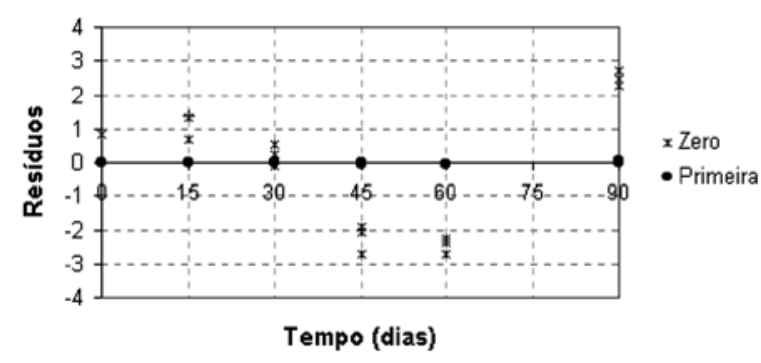

FIGURA 3 - Distribuição dos resíduos para os modelos de zero e de primeira ordem

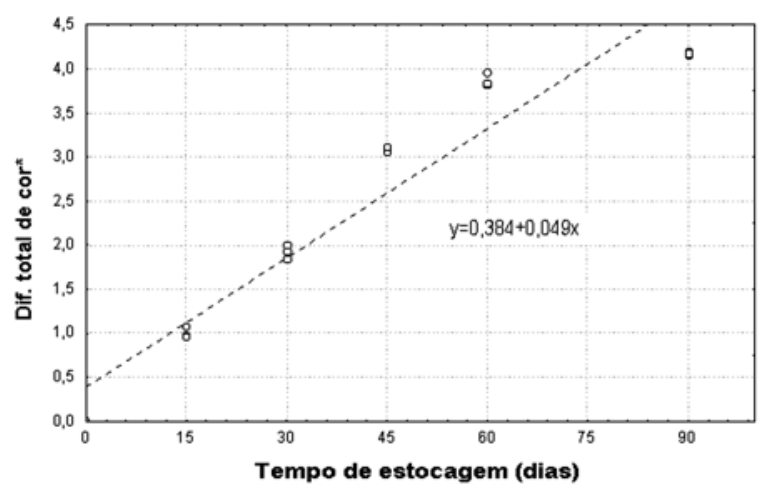

FIGURA 4 - Modelo cinético da diferença total de cor da polpa de pitanga congelada por 90 dias - ordem zero

\section{4 - Análise sensorial}

Os resultados obtidos na análise sensorial da polpa de pitanga servido na forma de néctar são mostrados na Tabela 5, e observou-se que a aparência foi o atributo mais afetado na qualidade da polpa de pitanga congelada ao final do tempo de estocagem.

TABELA 5 - Médias dos atributos cor, aroma e sabor do néctar de pitanga

\begin{tabular}{|c|c|c|c|}
\hline \multirow{2}{*}{$\begin{array}{c}\text { Tempo de } \\
\text { estocagem (dias) }\end{array}$} & \multicolumn{3}{|c|}{ Atributo } \\
\hline & Aparência & Aroma & Sabor \\
\hline 0 & $6,49 \pm 1,50^{\mathrm{a}}$ & $6,5 \pm 1,67^{a}$ & $6,28 \pm 1,97^{a, b}$ \\
\hline 30 & $6,52 \pm 1,68^{\mathrm{a}}$ & $6,99 \pm 1,78^{\mathrm{a}}$ & $7,05 \pm 1,56^{a}$ \\
\hline 60 & $6,28 \pm 1,69^{a}$ & $6,92 \pm 1,72^{\mathrm{a}}$ & $7,33 \pm 1,36^{\mathrm{a}}$ \\
\hline 90 & $3,52 \pm 1,76^{b}$ & $6,68 \pm 1,80^{a}$ & $5,65 \pm 2,40^{b}$ \\
\hline
\end{tabular}
estatistica $(p \leq 0,05)$.

Como pode ser observado na Figura 5, a porcentagem de provadores que provavelmente e certamente comprariam o produto foi de $51,28 \%, 74,35 \%, 63,33$ e $30 \%$, para os tempos 0, 30, 60 e 90 dias de estocagem, respectivamente. Ao mesmo tempo, verificou-se para as atitudes certamente e provavelmente não comprariam o produto valores de $10,26 \%, 7,69 \%, 10 \%$ e $53,33 \%$, para os tempos 0, 30, 60 e 90 dias de estocagem, respectivamente. 


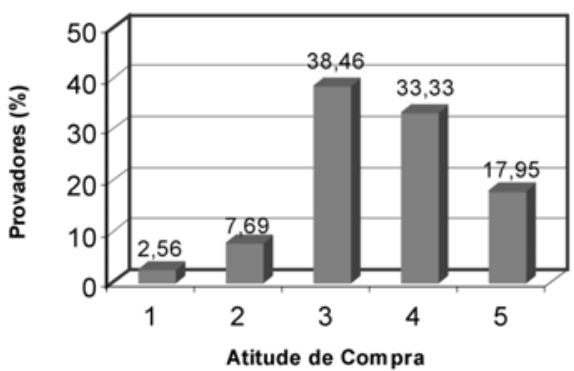

60 dias de estocagem

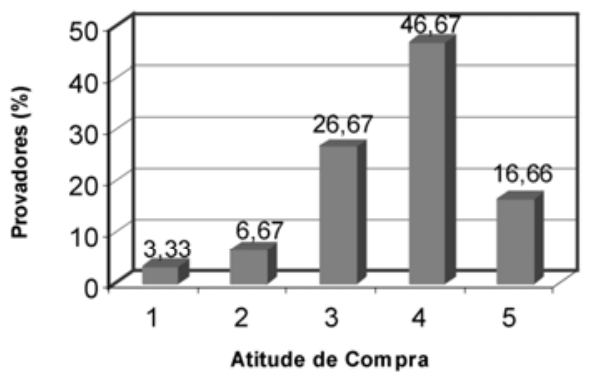

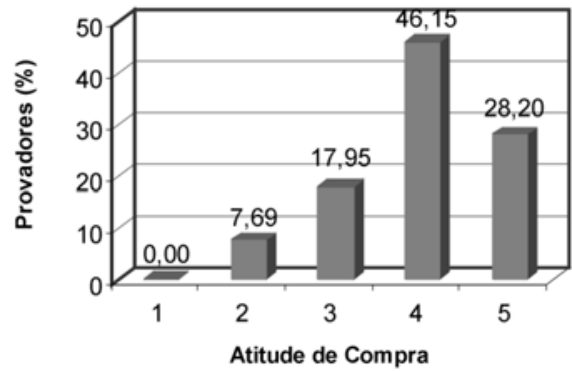

90 dias de estocagem

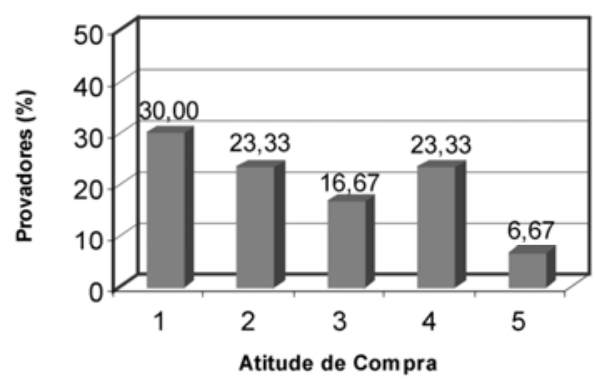

FIGURA 5 - Atitude de compra dos provadores para o néctar de pitanga durante os tempos de estocagem avaliados (0, 30,60 e 90 dias)

Diante destes resultados, concluiu-se que a polpa de pitanga após 90 dias de estocagem a $-18^{\circ} \mathrm{C}$ não apresentou boa aceitabilidade, principalmente pela mudança de cor e a separação parcial de fases observada no produto. A separação parcial de fases do produto (soro e polpa) é um sério defeito em polpas e sucos de frutas tropicais, e tem sido atribuída à ação da pectinametilesterase (PME) [27].

Diante disso, sugere-se à aplicação de tratamento térmico, como o branqueamento ou a pasteurização, para inativação completa das enzimas presentes na polpa de pitanga para garantir a estabilidade física, físicoquímica e sensorial dos produtos com ela formulados.

\section{4 - CONCLUSÕES}

- Após 90 dias de estocagem a-18 C, a polpa de pitanga manteve-se dentro dos padrões de identidade e qualidade exigidos pela legislação vigente.

- Houve um decréscimo significativo no teor de carotenóides totais da polpa de pitanga congelada, cerca de $13,76 \%$, nos primeiros 30 dias de estocagem; após esse periodo o teor desses pigmentos manteve-se praticamente inalterado.

- De acordo com a análise sensorial, o atributo aparência variou significativamente $(\mathrm{p} \leq 0,05)$ aos 90 dias de estocagem, devido à ocorrência de separação par- cial de fases e alteração de cor, ocasionando uma forte queda na aceitabilidade sensorial e na atitude positiva de compra do néctar formulado com a polpa de pitanga armazenada por 90 dias a $-18^{\circ} \mathrm{C}$. Por isso, é fundamental a aplicação de um tratamento térmico na polpa de pitanga antes do congelamento a fim de inativar as enzimas presentes e, conseqüentemente, proporcionar a manutenção da qualidade sensorial dos produtos formulados com essa polpa.

\section{5 - REFERÊNCIAS BIBLIOGRÁFICAS}

[1] AOAC. Official Methods of Analysis of AOAC International. 14. ed. Arlington: Sidney Williams (Ed.), 1984.

[2] AOAC. Official Methods of Analysis of AOAC International. 16. ed. Gaithersburg: Patricia Cunniff (Ed.), 1997.

[3] BARROS NETO, B. de; SCARMINIO, I. S.; BRUNS, R. E. Como fazer experimentos: pesquisa e desenvolvimento na ciência e na indústria. 2 ed. Campinas: Editora da UNICAMP, 2002. Cap. 5.

[4] BRASIL. Ministério da Agricultura e do Abastecimento. Instrução Normativa n 01, de 7 de janeiro de 2000. Diário Oficial da União. Brasília, 10 de janeiro de 2000. Anexo II.

[5] CAVAlCANTE, M. L. Composição de carotenóides e valor de vitamina A em pitanga (Eugenia uniflora L.) 
e acerola (Malpighia glabra L.). Rio de Janeiro, 1991. 86p. Dissertação de Mestrado, Instituto de Nutrição, Centro de Ciências da Saúdes, Universidade Federal do Rio de Janeiro.

[6] CAVAlCANTE, M. L.; RODRIGUEZ-AMAYA, D. B. Alteration of the carotenoid composition during manufacture and storage of frozen Eugenia uniflora fruit. In: 9th World Congress of Food Science and Technology. Budapeste, 1995.

[7] COSTELL, E.; DURAN, L. Sensory analysis in the quality-control of foods: 1. Introduction. Revista de Agroquímica y Tecnologia de Alimentos, Valencia, v. 21 , n. 1, p. 1-10, 1981

[8] FARIA, E. V de. Avaliação e determinação da vida-deprateleira: a importância da análise sensorial. In: MOURA, S. C. S. R. de; GERMER, S. P. M. Manual do curso reações de transformação e vida-de-prateleira de alimentos processados. Campinas: Instituto de Tecnologia de Alimentos, 2002. 75p.

[9] FU, B.; LABUZA, T. P. Shelf life of frozen foods. In: LABUZA, T. P.; FU, B. Shelf Life Testing: Procedures and Prediction Methods. Denver: CRC Press, 1997. Cap. 19. p.377-415.

[10] GOMES, R. P. Fruticultura brasileira. 2.ed. São Paulo: Nobel, 1975. 446p.

[11] GUIMARÃES, F. A.; HOLANDA, L. F. F.; MAIA, G. A.; MOURA FÉ, J. A. Contribuição tecnológica ao processamento e conservação da polpa de pitanga (Eugenia uniflora L.). Boletim da Sociedade Brasileira de Ciência e Tecnologia de Alimentos, Campinas, v. 17, n. 1, p. 01-13, 1983.

[12] LABUZA, T. P. Shelf-life dating of foods. Westport: Food and Nutrition Press, 1982.

[13] LEDERMAN, I. E.; BEZERRA, J. E. F.; CALADO, G. A pitangueira em Pernambuco. Recife: Empresa Pernambucana de Pesquisa Agropecuária - IPA, 1992. 20 p.

[14] LIMA, V. L. A. G. de; MÉlO, E. A.; LIMA, D. E. S. Fenólicos e carotenóides totais em pitanga. Scientia Agricola, Piracicaba, v. 59, n. 3, p. 447-450, 2002.

[15] LIMA, V. L. A. G. de; MÉLO, E. A.; LIMA, D. E. S. Efeito da luz e da temperatura de congelamento sobre a estabilidade das antocianinas da pitanga roxa. Revista de Ciência e Tecnologia de Alimentos, Campinas, v. 25, n. 1, p. 92-94, 2005.

[16] LOMAURO, C. J.; BAKSHI, A. S.; LABUZA, T. P. Evaluation of food moisture sorption isotherm equations. Part I: fruit, vegetable and meat products. Lebensmittel Wissenschaft und Technologie, London: Academic Press, v. 18, p. 111-117, 1985.
[17] MEILGAARD, M.; CIVILlE, G. V.; CARR, B. T. Sensory evaluation techniques. Boca Raton: CRC Press, 1999. $387 \mathrm{p}$.

[18] MAYER-MIEBACH, E.; SPIEß, W. E. L. Infuence of cold storage and blanching on the carotenoid content of Kintoki carrots. Journal of Food Engineering, Essex: Elsevier Applied Science, v. 56, p. 211-213, 2003.

[19] MÉlo, E. A.; LiMA, V. L. A. G.; NASCimento, P. P. Temperatura no armazenamento de pitanga. Scientia Agricola, Piracicaba, v. 57, n. 4, p. 629-634, 2000.

[20] Muñoz, A. M.; CIVIlle, G. V.; CARR, B. T. Sensory evaluation in quality control. New York: Van Nostrand Reinhold, 1992. 240p.

[21] RODRIGUEZ-AMAYA, D. B. Carotenoids and food preparation: the retention of provitamin $A$ carotenoids in prepared, processed and stored foods. Washington, DC: OMNI/USAID, 1997.

[22] RODRIGUEZ-AMAYA, D. B. A guide to carotenoid analysis in foods. Washington: ILSI Press, 1999. 64p.

[23] STATSOFT, INC. (1995). STATISTICA for Windows [Computer program manual]. Tulsa, OK: StatSoft, Inc., 2325 East 13th Street, Tulsa, OK 74104, (918) 583-4149, fax: (918) 583-4376.

[24] STONE, H.S.; SIDEL, J.L. Sensory Evaluation Practices. 2 ed. San Diego: Academic Press, 1993. 336p.

[25] TAOUKIS, P. S.; LABUZA, T. P.; SAGUY, I. S. Kinetics of food deterioration and shelf-life prediction. In: VALENTAS,ÊK. J.; ROTSTEIN,ÊE.; SINGH,ÊR. P. The handbook of food engineering practice. Boca Raton:ÊCRC Press LLC, 1997. p.361-402.

[26] THAKUR, B. R.; ARYA, S. S. Relative suitability of plastic films for the frozen storage of mango pulp. Journal of Food Processing and Preservation, Westport v. 12, n. 3, p. 171-178, 1988.

[27] VERSTEEG, C.; ROMBOUTS, F. M.; SPAANSEN, C. H.; PILNIK, W. Thermostability and orange juice cloud destabilizing properties of multiple pectinesterases from orange. Journal of Food Science, Chicago, v. 45, n. 4, p. 969-971, 1980.

\section{6 - AGRADECIMENTOS}

Os autores agradecem ao Conselho Nacional de Desenvolvimento Científico e Tecnológico (CNPq) pelo apoio financeiro recebido. Às técnicas responsáveis pelo Laboratório de Frutas, Hortaliças e Produtos Açucarados do DTA-FEA-UNICAMP Ana Koon e Priscila Albertini. 